\title{
Track monitoring from the dynamic response of a passing train: a sparse approach
}

\author{
George Lederman ${ }^{\mathrm{a}, \mathrm{b}, *}$, Siheng Chen $^{\mathrm{b}}$, James H Garrett ${ }^{\mathrm{a}}$, Jelena Kovačevićc,c ${ }^{\mathrm{b}}$, Hae Young \\ Noh $^{\mathrm{a}, \mathrm{b}}$, Jacobo Bielak ${ }^{\mathrm{a}}$ \\ ${ }^{a}$ Civil and Environmental Engineering, Carnegie Mellon University \\ ${ }^{b}$ Electrical and Computer Engineering, Carnegie Mellon University \\ ${ }^{c}$ Biomedical Engineering, Carnegie Mellon University \\ Pittsburgh, PA 15213 USA
}

\begin{abstract}
Collecting vibration data from revenue service trains could be a low-cost way to more frequently monitor railroad tracks, yet operational variability makes robust analysis a challenge. We propose a novel analysis technique for track monitoring that exploits the sparsity inherent in train-vibration data. This sparsity is based on the observation that large vertical train vibrations typically involve the excitation of the train's fundamental mode due to track joints, switchgear, or other discrete hardware. Rather than try to model the entire rail profile, in this study we examine a sparse approach to solving an inverse problem where (1) the roughness is constrained to a discrete and limited set of "bumps"; and (2) the train system is idealized as a simple damped oscillator that models the train's vibration in the fundamental mode. We use an expectation maximization (EM) approach to iteratively solve for the track profile and the train system properties, using orthogonal matching pursuit (OMP) to find the sparse approximation within each step. By enforcing sparsity, the inverse problem is well posed and the train's position can be found relative to the sparse bumps, thus reducing the uncertainty in the GPS data. We validate the sparse approach on two sections of track monitored from an operational train over a 16 month period of time, one where track changes did not occur during this period and another where changes did occur. We show that this approach can not only detect when track changes occur, but also offers insight into the type of such changes.
\end{abstract}

Keywords: Signal Processing, Sparse Representation, Orthogonal Matching Pursuit, Vehicle-based Inspection, Inertial Sensing

\footnotetext{
*Corresponding author

Email addresses: george.lederman@gmail.com (George Lederman), sihengc@andrew.cmu.edu (Siheng Chen), garrett@cmu.edu (James H Garrett), jelenak@cmu.edu (Jelena Kovačević), noh@cmu.edu (Hae Young Noh), jbielak@cmu.edu (Jacobo Bielak)
} 


\section{Introduction}

Monitoring track geometry is essential to ensuring the safe operation of rail-infrastructure, yet current inspection techniques require track downtime $[1,2]$. Monitoring railroad track geometry with in-service vehicles could reduce required track downtime while providing more continuous information than using dedicated inspection vehicles $[3,4,5,6,7]$. Decreases in the cost of sensing equipment have made monitoring tracks from in-service vehicles economical while recent advances in analytical techniques have made processing the collected data feasible.

Three main types of sensing technology have been proposed for in-service trains: optical sensors (using lasers) [8, 9], magnetic flux sensors (also called Foucault currents or Eddy Currents) $[10,11]$, and inertial sensors (using accelerometers) $[3,4,5,6,12,13,14,15$, $16,17,18,19,20]$. Optical sensors are used widely on track geometry cars, however they stop functioning if the lens becomes dirty. They require constant cleaning and maintenance, and thus are not appropriate for long term-monitoring from in-service vehicles [21]. Foucault current monitoring requires a magnetic coil placed close to the rail, making the coil vulnerable to objects along the track. Given the drawbacks of other sensing technologies, inertial sensors have become the most popular approach. The sensors are often placed on the axle box $[3,4,5]$, but can be placed anywhere on the train even inside the cabin [18]. The challenge in using accelerometers lies in analyzing the collected data.

There have traditionally been two approaches to analyzing train-based accelerometer data: using an explicit model or an implicit model. Explicit models attempt to determine the precise track profile, typically using a priori knowledge of the train and its suspension system to solve an inverse problem $[15,18]$. For each pass over the tracks, the track profile is estimated; deterioration in the tracks can be detected as changes in the profile. Implicit models derive a feature from the accelerometer data which often serves as a proxy for track geometry or roughness. In these models, deterioration is detected when the values of the features change. Notable implicit models have been based on wavelets [3, 12, 22, 23], the Short Time Fourier Transform [4], signal standard deviation [5], and signal energy [6, 24].

Implicit models tend to be more robust and may allow for the detection of a change, but as the feature is not directly representative of the track state, they often do not provide insight into the nature of the change. Explicit models can estimate the track profile before and after a change so that the mechanism of the change could in theory be determined. However, to our knowledge, this technique has not been used for long-term monitoring $[15,18]$; the instability in solving the inverse problem directly makes consistently determining the track profile a challenge.

In this paper, we propose a novel approach to approximate the track profile. As the dynamic response of the train contains information both about the train and about the tracks themselves, we decompose the vibration signal in an effort to separate these sources. To do so, we solve an inverse problem which determines the train's main dynamic properties and the profile of the track.

Solving such an inverse problem can be unstable; we constrain the problem to make it tractable. The first constraint comes from the observation that the train's suspension is 
typically activated by a few large bumps in the track. Thus we aim to find these discrete "bumps" and do so by enforcing sparsity in the estimated track profile.

The second constraint pertains to the characterization of the train system. We know the approximate properties of the train a priori, but these can change over time depending for example, on passenger loading. Thus we want to solve for train properties from the data, but doing so directly can provide noisy results. In a previous study [6], we found that the train's fundamental mode dominates its response. So we limit the problem to characterizing this fundamental mode which can be represented as a single degree-of-freedom damped oscillator. By modeling one part of the train vibration, we are able to reduce some of the train dependent variables in the signal.

In some ways, this technique resembles the explicit model mentioned earlier. We solve an inverse problem that provides information about both the train and the tracks. However, due to the sparse constraints, the resultant track profile is quite different from the true profile. In this regard, the technique resembles the implicit model more closely. The resultant sparse track profile can be thought of as a feature indicative of track state. We can explore the merit of this sparse constraint by testing how closely changes in the sparse profile match changes in true track profile. Compared to other features, like wavelets, the sparse profile provides greater insight because it can show the direction in which the track has changed, for example either settlement or uplift.

Enforcing sparsity in the track has a number of benefits [25, 26, 27, 28]: (1) the problem is constrained so some properties of the train system can be found without making the problem ill-posed, (2) the discrete bump locations can be used to locate the train, overcoming GPS error, and (3) the size of the bumps are useful low-dimensional features for detecting the significance of changes in the track.

(1) We characterize the train system while constraining it to the physics of the problem. We require that the transfer function correspond to a simple damped oscillator. When enforcing this condition, the parameters found relate to the stiffness and damping ratio of the main suspension between the wheel truck and the train chassis. This makes physical sense because when a large bump in the tracks excites the train, the largest displacement is in the primary suspension, and we require that the approximate roughness models only large bumps. Unlike previous methods in which the parameters of the train must be known a priori, our approach solves for the train properties in the process of solving for the track profile.

(2) We locate the train using a GPS antenna, but due to overhead interference and other factors, the position-error can exceed $10 \mathrm{~m}$. This level of error makes it challenging to compare data between passes. Train localization has been studied in the literature primarily for collision avoidance [29]. Some researchers have proposed using track features to help localize trains in this context [30, 19]. For monitoring purposes, precise localization is of paramount importance. Enforcing sparsity facilitates localization because the position of the train can be found relative to the sparse bumps.

(3) Finally, the size of the bumps can be used to determine whether the tracks have changed or deteriorated. If a complete rail profile were to be calculated, the 
high dimensionality of the data (proportional to track length) would make robust change detection more challenging; the low-dimensionality of bump height as a feature simplifies change detection, as will be shown in Section 4.

We show the application of this sparse approach on data collected from a light-rail vehicle over a 16-month period of time in Pittsburgh, PA. We explore the consistency of the method over time, both in terms of identifying the parameters of the train and the track profile. We study how consistently the proposed approach can identify the same bumps in the track, both when the tracks remain unchanged over a period of time, and when the tracks change due to deterioration or maintenance.

\section{Problem Description}

As the train travels along the tracks, the suspension filters the roughness of the track. Let $u$ be the vertical displacement of the train, and $r$ be the vertical track profile. If the train suspension is modeled as linear ${ }^{1}$, the relationship between the acceleration of the train $\ddot{u}$ and the track profile $r$ can be written as

$$
\ddot{u}=h * r \text {. }
$$

Here $h$ is the impulse acceleration response of the train system, and $*$ is the convolution operator. $\ddot{u}, r$, and $h$ are all $p$-dimensional vectors corresponding to $p$ points in time. Such time-based sampling is typical when recording values with digital equipment. However, because the vector $r$ is sampled at constant time intervals, the distance between points varies if the train's speed is not constant (this is discussed in more detail in the following section).

If $h$ and $r$ are known, we refer to Eq. 1 as the forward formulation for evaluating $\ddot{u}$. On the other hand, if $h$ and $\ddot{u}$ are known but $r$ is not, Eq. 1 can be considered an inverse problem in which the roughness profile can be found through deconvolution. In the frequency domain (denoted by the hat symbol), this is written as

$$
\hat{r}=\frac{\hat{\ddot{u}}}{\hat{h}} .
$$

Most studies that try to model the track profile explicitly use this inverse formulation.

Real et al. [15] estimate the transfer function $\hat{h}$ by modeling the train as a two degree-offreedom oscillator. Directly solving Eq. (2) can be unstable as the transfer function $\hat{h}$ of an oscillator approaches zero for high frequencies while the measured data $\hat{\ddot{u}}$ can have non-zero values at high frequencies. This potentially leads to amplification of high-frequency data. Real et al. address this instability by bandpass filtering the measured data. The challenge is how to define an appropriate bandpass filter; in their paper, they fit a filter using one

\footnotetext{
${ }^{1}$ The train's suspension is not purely linear; for example, the interface between the wheel and the rail is often modeled as a non-linear Hertzian spring, but for the vast majority of analyses, this slight non-linearity can be approximated as linear [31].
} 
section of track, but that filter appears not to work well on other track sections. That the filter is not generalizable could be explained by the fact that the train system is more complicated than the two degree-of-freedom model they use. Thus the true system response has some non-zero values at high frequency, and the filter is an attempt to compensate for an oversimplified model for $\hat{h}$.

O'Brien et al. [18] use a different approach to avoid the instability inherent in dividing by the transfer function. They use the cross-entropy method to generate a family of roughness profiles, then use the forward formulation in Eq. (1) to calculate the response that the roughness would generate. They then repeat the process, each time attempting to minimize the mean squared error between the generated response and the measured response. This iterative process is computationally expensive requiring several hours of processing to analyze a simulated $20 \mathrm{~m}$ section of track. Furthermore, this approach has not yet been tested on operational data, and its sensitivity to noise has not yet been studied.

In our approach, we aim to preserve the computational efficiency of directly solving the inverse problem while constraining the problem sufficiently so that a stable solution is found even if the exact transfer function is not known. Without knowledge of the precise transfer function we cannot find the precise track profile, but as a trade-off, we aim to find only the location and magnitude of the "bumps" or irregularities along the track. As will be described in the following section, we achieve this by enforcing sparsity.

\section{Algorithm}

We want to find the train's transfer function and the track roughness that best approximate the measured vertical accelerometer data. This can be written as

$$
\min _{h, r}\|\hat{\ddot{u}}-\hat{H} \hat{r}\|_{2}
$$

where $\|\cdot\|_{2}$ is the $\ell_{2}$ norm, $\hat{\ddot{u}}$ and $\hat{r}$ are $p$-dimensional vectors corresponding to the $p$ observations, and $\hat{H}$ is a $p \times p$ diagonal matrix of the transfer function,

$$
\hat{H}=\left[\begin{array}{cccc}
\hat{h}[1] & 0 & \ldots & 0 \\
0 & \hat{h}[2] & \ldots & 0 \\
\vdots & \vdots & \ddots & \vdots \\
0 & 0 & \ldots & \hat{h}[p]
\end{array}\right]
$$

Without constraints, this problem is ill-posed and has trivial solutions: for example, $\hat{H}$ could be the identity matrix and $\hat{r}=\hat{\ddot{u}}$, which would lead to a perfect solution. But nothing would be learned either about the nature of the track roughness or about the train system. Instead we constrain the track profile to a set of $n$ discrete bumps and the transfer function to model only the train's fundamental mode. This is achieved by limiting the transfer function to a single degree-of-freedom oscillator, seen in Fig. 1, where the range of possible parameters for stiffness, mass, and damping are chosen to match the range of values the train can have. It is important to note that the train has more than one mode. However, the first mode 
appears to be the most prominent [6]. By constraining the transfer function to model a single mode, we imbue physical meaning into the solution of the inverse problem.

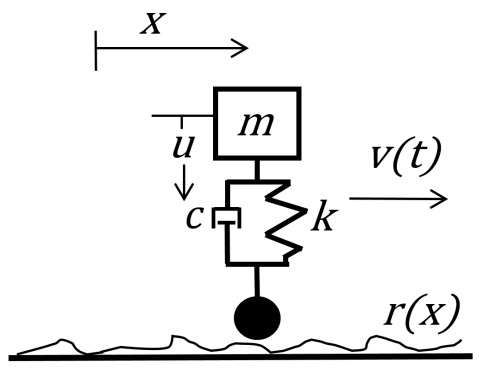

Fig. 1: A representation of the train as a single degree-of-freedom oscillator

The vertical motion of the oscillator is governed by spring stiffness, $k$, damping, $c$, vehicle mass, $m$, and the roughness experienced by the train $r(t)$,

$$
m \ddot{u}(t)+c \dot{u}(t)+k u(t)=c \dot{r}(t)+k r(t) .
$$

Here $r(t)$ can be found by interpolating $r(x)$, the actual roughness profile (in the spatial domain), according to the train's position,

$$
r(t)=\left.r^{\prime}(x)\right|_{x(t)} .
$$

In the frequency domain, Eq. (5) can be written as

$$
-m \omega^{2} \hat{u}(\omega)+\operatorname{ci\omega } \hat{u}(\omega)+k \hat{u}(\omega)=\operatorname{ci\omega } \hat{r}(\omega)+k r(\omega),
$$

which can be rearranged to define the transfer function, $\hat{h}$,

$$
\hat{h}(\omega)=\frac{\hat{\ddot{u}}(\omega)}{\hat{r}(\omega)}=(i \omega)^{2} \frac{i \omega c+k}{-\omega^{2} m+i \omega c+k},
$$

where $\omega$ is frequency and $i=\sqrt{-1}$. It is often more intuitive to consider the physical properties of the train in terms of natural frequency, $w_{n}=\sqrt{k / m}$, and damping ratio, $\zeta=c / 2 m \omega_{n}$, which allows the train parameters to be described with two values (natural frequency and damping ratio), instead of three (mass, stiffness and damping),

$$
\hat{h}(\omega)=(i \omega)^{2} \frac{2 i \zeta \omega_{n} \omega+\omega_{n}^{2}}{-\omega^{2}+2 i \zeta \omega_{n} \omega+\omega_{n}^{2}} .
$$

To find a discrete representation of $\hat{h}$, we evaluate Eq. (9) at discrete frequencies, $\omega[p]$, producing discrete values of the transfer function, $\hat{h}[p]$. Returning to Eq. (3), we can now 
write the physical constraints we will enforce,

$$
\begin{array}{ll}
\min _{h, r} & \|\hat{\ddot{u}}-\hat{H} \hat{r}\|_{2}, \\
\text { subject to } & \|r\|_{0} \leq n \\
& \hat{h}[p]=(i \omega[p])^{2} \frac{2 i \zeta \omega_{n} \omega[p]+\omega_{n}^{2}}{-\omega[p]^{2}+2 i \zeta \omega_{n}^{2} \omega[p]+\omega_{n}^{2}},
\end{array}
$$

where $n$ is the number of sparse bumps and $\|\cdot\|_{0}$ denotes the $\ell_{0}$ norm, defined as the the number of non-zero elements within the vector. In this case the constraint limits the roughness profile, $r$, to having at most $n$ non-zero elements. Here the discrete frequencies, $\omega[p]$, should match the frequencies of the collected data after taking the Fourier transform. This means it depends on the sampling rate and duration of the collected data. For example, if we apply this sparse approach to 1 second of data sampled at $1.6 \mathrm{kHz}, \mathrm{w}[\mathrm{p}]$ would be the corresponding frequency range, $-799 \mathrm{~Hz}$ to $800 \mathrm{~Hz}$ in $1 \mathrm{~Hz}$ increments.

In minimizing Eq. (10), we find a sparse version of the track profile due to the $\ell_{0}$ constraint. In addition, the properties of the identified damped oscillator characterize one of the trian's modes, typically the fundamental mode.

Directly solving this minimization problem would be computationally expensive; instead of simultaneously solving for the optimal transfer function and roughness, we solved for each iteratively. We used an Expectation Maximization approach [32] to first solve for the transfer function then the roughness, repeating until convergence.

As the first step, we create a dictionary of possible transfer functions based on a priori knowledge of the train's fundamental mode. Each transfer function is a $p \times 1$ vector, found by selecting values for the damping ratio and the natural frequency, then solving the equation from the second constraint shown in Eq. (10). Here the discrete frequency values, $\omega[p]$, correspond to the discrete frequencies of $\hat{\ddot{u}}$. We place all vectors into a dictionary matrix $\hat{D}_{h}$, then solve

$$
\begin{array}{ll}
\min _{\alpha} & \left\|\hat{\ddot{u}}-\hat{R} \hat{D}_{h} \alpha\right\|_{2}, \\
\text { subject to } & \|\alpha\|_{0} \leq 1,
\end{array}
$$

where $\hat{R}$ is a diagonal matrix of the roughness profile in the frequency domain and $\alpha$ is a vector indicating the selected transfer function from the dictionary. If this is the first iteration, an arbitrary roughness can be used initially; for this roughness, we used $n$ evenly spaced bumps. If this is not the first iteration, the roughness found in the second step is used. Given the $\ell_{0}$ constraint, directly solving the problem is $N P$-hard [33] so we use orthogonal matching pursuit (OMP [34]), a computationally efficient way to select the optimal $\alpha$. In this case $\alpha$ has one non-zero element; the location of this non-zero element within the vector corresponds to the location of the best transfer function within the dictionary, $\hat{D}_{h}$, which is the transfer function that minimizes the $\ell_{2}$ norm. The value of the non-zero element is 
found using least squares minimization (thus is not necessarily an integer). We then update the transfer function using $\hat{h}=\hat{D}_{h} \alpha$.

As the second step, we create a dictionary of the possible roughnesses. We want to allow the solution to have a discrete bump at any point along the signal, so our dictionary is a collection of all the possible vectors with a single non-zero value. The result is the identity matrix. Because the calculation is done in the frequency domain, the dictionary is the Fourier transform, $\mathcal{F}$, of the identity matrix, $I: \hat{D}_{r}=\mathcal{F}(I)$ which is known as the discrete Fourier transform matrix [35]. We compute

$$
\begin{array}{ll}
\min _{\beta} & \left\|\hat{\ddot{u}}-\hat{H} \hat{D}_{r} \beta\right\|_{2}, \\
\text { subject to } & \|\beta\|_{0} \leq n,
\end{array}
$$

where $\beta$ is the vector indicating the selected roughness bumps from the dictionary and $\hat{H}$ is the diagonalization of the transfer function, $\hat{h}$, found in the first step. We solve this problem using OMP as before [34]. In this method, first the best bump (i.e. the bump that minimizes the $\ell_{2}$ norm) is selected, then the second best bump is selected, and the magnitude of each adjusted using least squares minimization. This process is repeated up to the $n$ allowed bumps. While the first bump is guaranteed to be optimal, the combination of bumps is not necessarily optimal. Given the results we have achieved, this approach appears adequate for the task, and, as discussed previously, finding the optimal solution is not practical as the problem is $N P$-hard. The advantage of not necessarily selecting the optimal combination of bumps is that the process is fast computationally.

Having determined $\beta$, we update the roughness as $\hat{r}=\hat{D}_{r} \beta$. We then return to the first step and repeat the process iteratively until convergence. Note that this process is expected to converge because in each step the $\ell_{2}$ norm error either decreases or stays the same.

\section{Validation on Operational Data}

We apply our sparse approach to a large field data-set collected from an in-service lightrail vehicle. In this section, we explore how well the sparse approach can analyze noisy data and detect changes in rail infrastructure.

\subsection{Description of operational data collection}

We collected vibration data from an in-service light-rail vehicle in Pittsburgh, PA over a 16-month period in partnership with the Port Authority of Allegheny County. When we instrumented the vehicle, we were interested in whether we could monitor the rail infrastructure from sensors on the wheel truck as well as sensors inside the cabin. Cabin-based sensors are isolated from the tracks by the train's suspension, so the signal-to-noise ratio is lower. However, since they are less vulnerable and more easily accessible, such sensors contribute to lower operational and maintenance costs of the sensing system itself. Two uni-axial accelerometers, Vibrametric Model 5102, were mounted inside the cabin as shown in Fig. 2a, and a tri-axial accelerometer, PCB 354C02, was mounted on the central wheel 


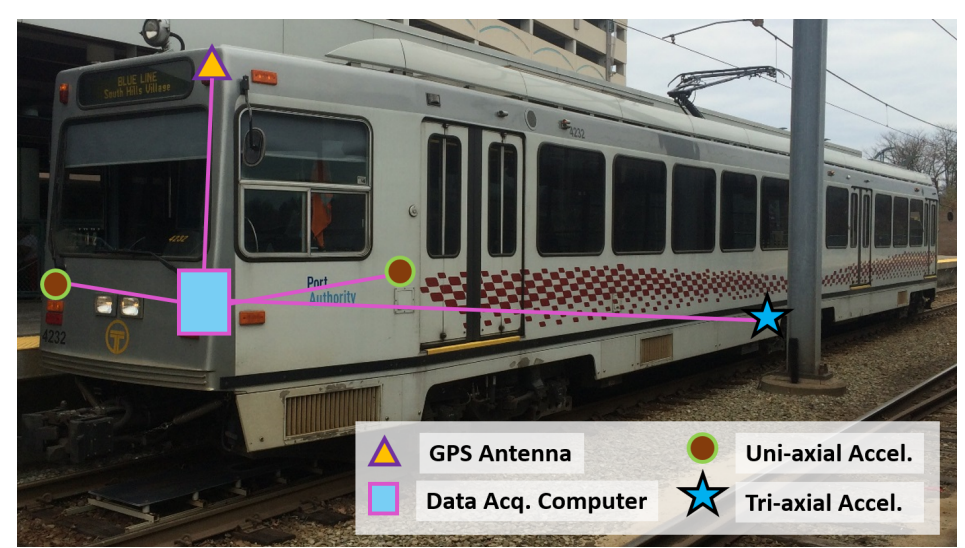

(a) Locations of the deployed sensors

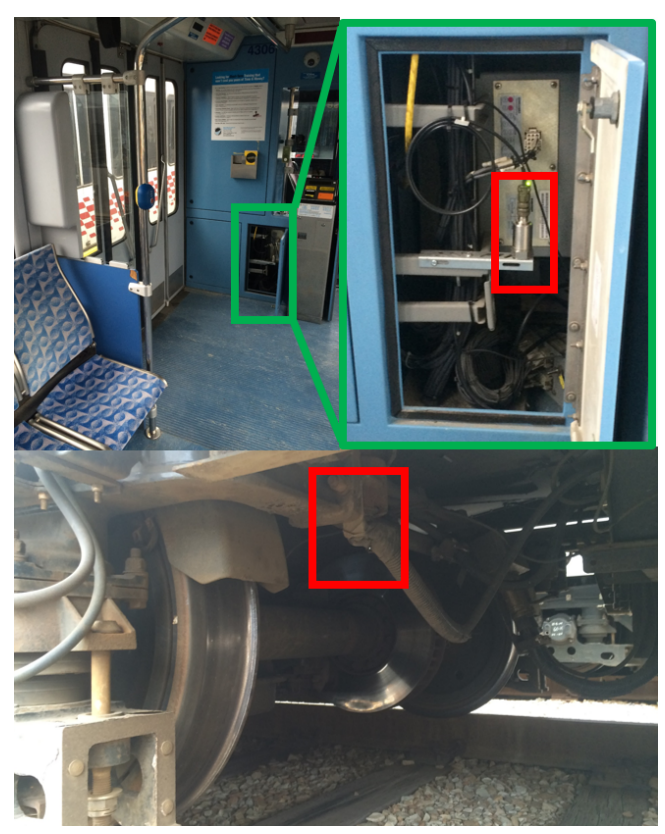

(b) Details of the sensors

Fig. 2: Instrumentation details. (a) Schematic of the data acquisition system. (b) Close-up details of sensors inside the train (above), and on the wheel axle (below). The Fig. on the top right shows details of the open cabinet (green box) and the red box highlights the sensor itself. The lower right picture shows the axle of the train with the tri-axial sensor highlighted by the red box.

truck (the central wheel truck is not powered so has less electrical noise). Both types of sensors can be seen in Fig. 2b. We also have a BU-353 GPS antenna just under the roof and an industrial grade data-acquisition computer. We sampled from the accelerometers at $1.6 \mathrm{kHz}$ and from the GPS at $1 \mathrm{~Hz}$. As in our previous work on this data-set, we focus on analyzing data from the accelerometers inside the train's cabin [6]. The train has a fundamental natural frequency of around $1.25 \mathrm{~Hz}$, found by analyzing the spectrum of the collected data; we estimated the damping ratio to be around 0.25 , a value confirmed by the literature [36]. More information on the instrumentation can be found in [37].

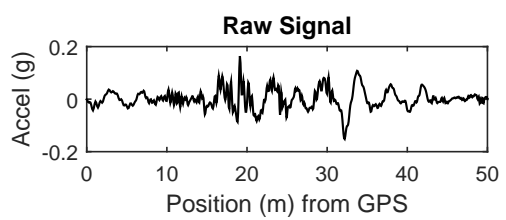

(a)

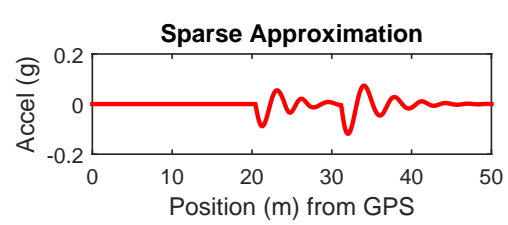

(b)

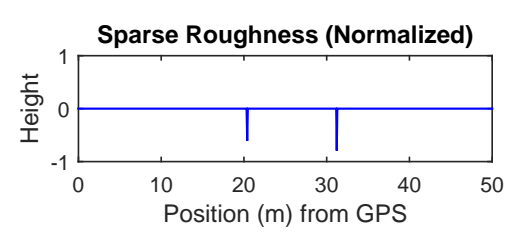

(c)

Fig. 3: Application of the sparse approach to raw signals, showing the found sparse bumps in (c). The sparse approximation shown in (b) can be thought of as the convolution of the train's transfer function with these sparse bumps. 

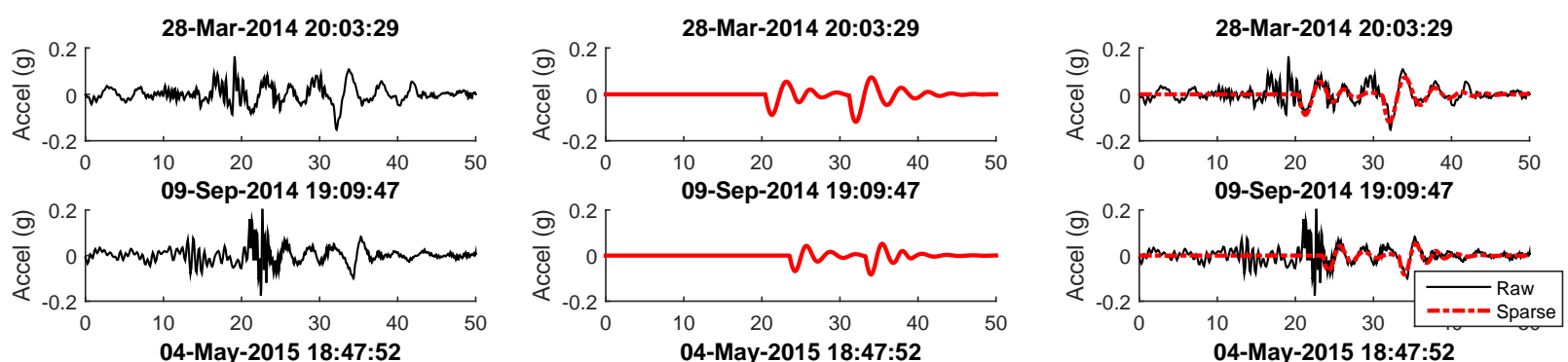

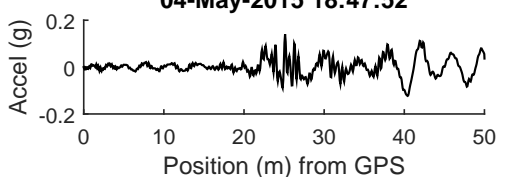

(a)

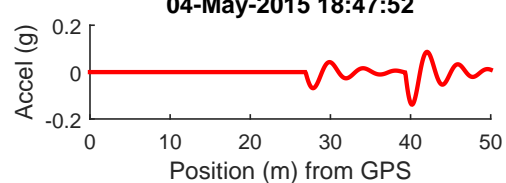

(b)

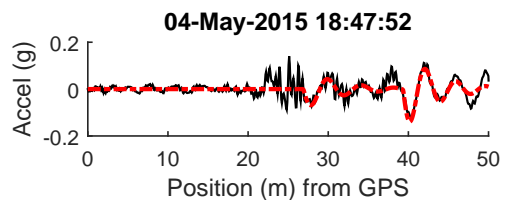

(c)

Fig. 4: Raw signal and sparse approximations for three passes of the train over a 50m section of track. (a) Raw signal from an accelerometer inside the cabin. (b) Sparse approximation of the signal. (c) Raw signal and sparse approximation overlaid.

\subsection{Application of the Sparse Approach to Operational Data}

Data from one pass of the train traversing a $50 \mathrm{~m}$ section of track is shown at the top of Fig. 3. The vibration signal is plotted as a function of the train's position according to GPS. The signal (Fig. 3a) is approximated using a sparse roughness profile with two bumps (Fig. 3b) leading to the sparse approximation of the track in (Fig. 3c).

For the dictionary of the transfer function, we choose a range of damping ratios from 0.15 to 0.4 in increments of 0.01 , and natural frequencies from $1 \mathrm{~Hz}$ to $1.75 \mathrm{~Hz}$ in increments of $0.01 \mathrm{~Hz}$. The algorithm then selects the best oscillator from this dictionary which included 1976 possibilities (26 damping ratios $\times 76$ frequencies). These ranges correspond to the approximate values of the natural frequency and damping ratio we had observed as well as the expected values of train primary suspension parameters documented in the literature $[6,36]$.

Three signals approximated in a similar fashion are shown in Fig. 4. Each has the same distinctive pattern, but the signals are not well aligned to one another due to GPS error. Again we apply the sparse approach to each signal, limiting the solution to two bumps, then iterating to convergence. Here two bumps are chosen based on the empirical observation that the train over this length of track experiences large amplitude excitation twice; if $n=1$, one or the other of the two bumps is selected; if $n>2$, the solution is less consistent with the additional bump modeling different parts of the signal with each pass. The sparse approximations of the signals are shown in Fig. 4b. Note the similarity between the sparse signals; this is because the train travels over the same bumps with each pass, so it has a similar response. The sparse approximation effectively denoises the signal to find only the response to these most significant bumps.

The sparse solution overlaid on the raw signal (Figure 4c) lends insight into which part of the signal the sparse solution approximates most closely. Note that the sparse solution does not approximate the raw signal when the train is first excited, but rather has a sort of 

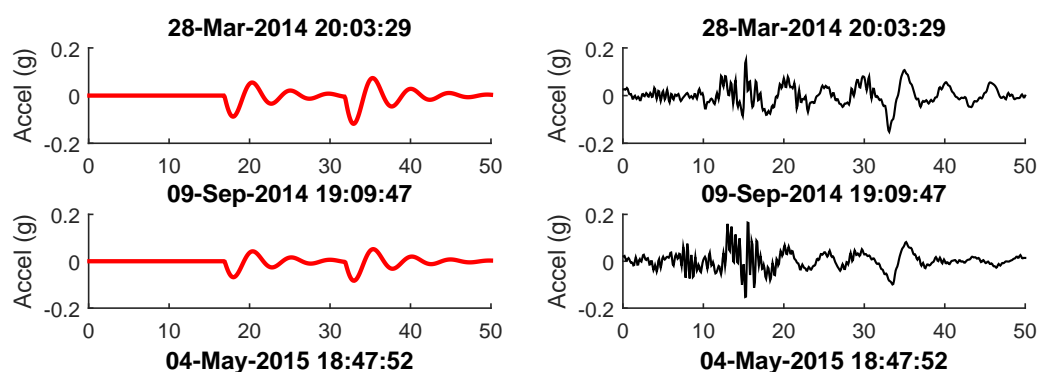

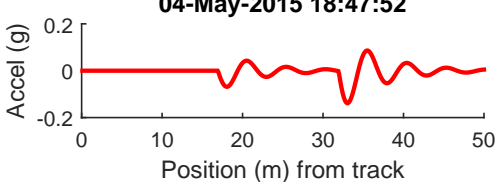

(a)

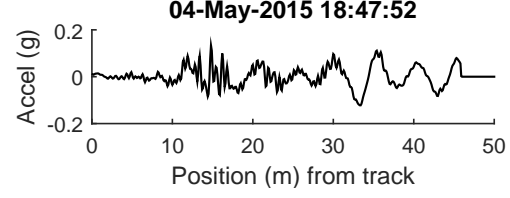

(b)

Fig. 5: Aligned sparse and raw signal. Using the discrete bumps found through the sparse approach, (a) the sparse approximation and (b) the raw signal can be aligned.

lag and approximates the latter part of the response to this excitation. When the train is first excited by a rough patch of track, the signal is noisy and depends on the shape of the roughness. After the initial excitation, the train's response more closely follows the response of a damped oscillator; the properties of this segment of the signal depend largely on the parameters of the train. As the sparse solution tries to decompose the signal into track roughness and the train's response, it approximates the sections when the train's vertical movement most closely resembles free-vibration.

In essence, our sparse approach uses the latter part of a vibration event to characterize the train's dynamic properties, where the train is the system of interest. Although to our knowledge this is the first time this concept has been used in vehicle-based monitoring, the concept has parallels in other domains. In seismology, the last part of a ground-motion signal, known as a coda wave, can be used to characterize geological structure, which can be thought of as a system in that context [38].

Returning to the monitoring problem, the location of the identified bumps in the track can be used to align the signals as shown in Fig. 5. This is accomplished by piecewise linear interpolation of the data so that the discrete bumps (shown in Fig. 3c) occur at the same location for each pass. For these three passes, each looks similar after alignment meaning the sparse approach has selected the same two bumps in the track from the signal (these are the bumps which minimize error). However, it is not guaranteed that the same two bumps will be selected from every signal; if different bumps are selected, alignment based on the bumps does not have a physical meaning. Such is the case with two of the three passes shown in Fig. 6.

As discussed earlier, the sparse approach tends to model the train's free vibration response after an excitation event. However, if the excitation itself happens to be similar to the free vibration response (i.e. the excitation appears to be the first oscillation in a series of damped oscillations), then the selected sparse bumps are not consistent with the bumps 

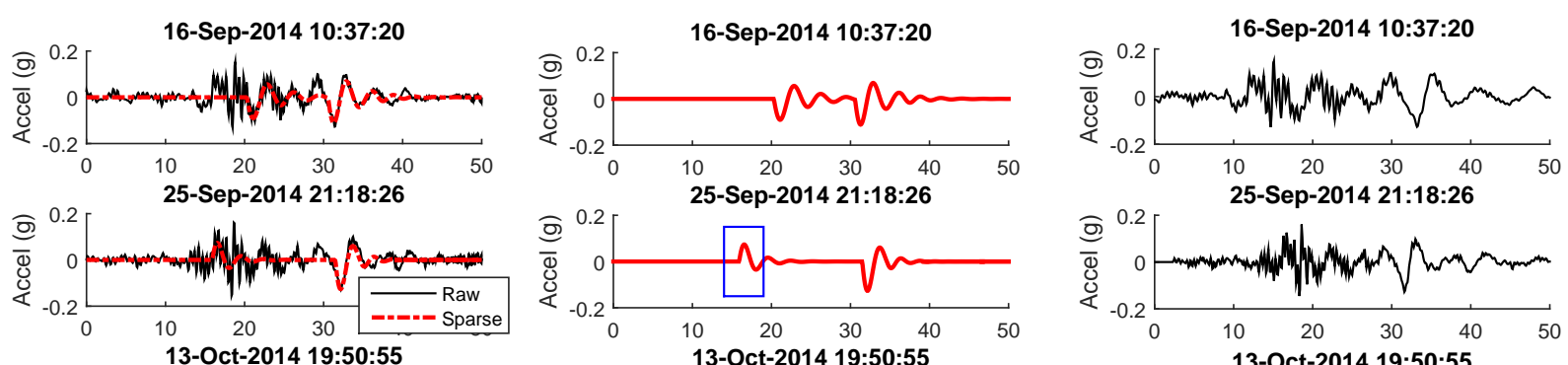

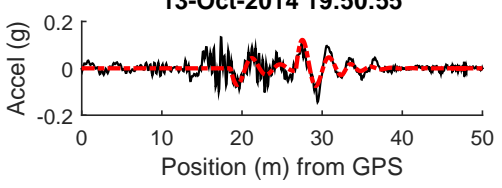

(a)

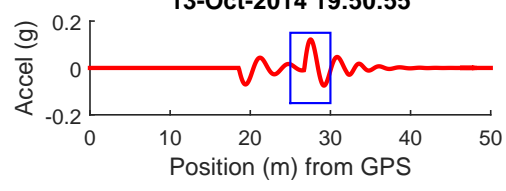

(b)

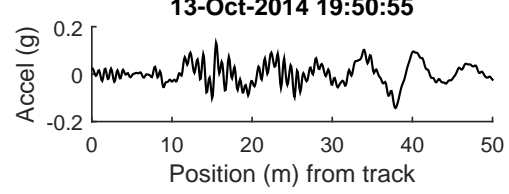

(c)

Fig. 6: An example of the sparse approach finding inconsistent bumps. (a) The raw signal and the sparse approximation overlaid. The sparse approximation in the first pass (16-Sep) matches the pattern in Figs. 4 and 5. The second and third passes each follow their own patten. (b) The sparse approximation with boxes to highlight where the sparse approximation differs. In Figs. 4 and 5 the sparse damped oscillations start downward; the two boxed oscillations start upward. (c) Alignment of the raw signal using the sparse approximation. Because the selected bumps are inconsistent, alignment due to track features is no better than the original GPS alignment.

found in similar signals. In Fig. 6, the sparse approximation of the 16-Sep signal matches the sparse approximation in Figures 4 and 5. For the 25-Sep signal, the first of the two sparse bumps fits the excitation rather than just the free vibration component. A similar phenomenon happens for the second bump in the 13-Oct signal. In Figures 4 and 5, the excitation is upward (positive), but the free-vibration response begins as the train accelerates downward (negative). The anomalous sparse vibrations in Fig. 6 appear upwards, as is highlighted by the blue boxes in Fig. 6b. The sparse approximation could differ either because of an issue with the approximation itself, for example, approximating the noise in the signal, or because of a variation in the train response, for example, the initial condition of the train before hitting a bump could affect its response. In either case, if inconsistent bumps are chosen by the sparse method, using these bumps for alignment leads to misaligned signals in Fig. 6c.

Fortunately, this inconsistency is easy to detect, and we can use the size and direction of the found sparse bumps. Fig. 7a shows the bump heights for all 267 passes recorded between February 2014 and June 2015. Note that while solving the inverse problem, the transfer function could be very small and the bumps very large, or vice versa, so when comparing values between solutions, we normalize the bump heights by setting the length of the found roughness vector to $1\left(\sum r^{2}=1\right)$. While the majority of signals have negative values for both of the bump heights, there are exceptions which follow the patterns explained in Fig. 6. Using a simple threshold, we can select only the passes with a negative first bump, and a second bump that is more negative than the first. Doing so leaves us with the 145 passes shown in Fig. 7b. Note here the track state appears to be consistent over time; this 


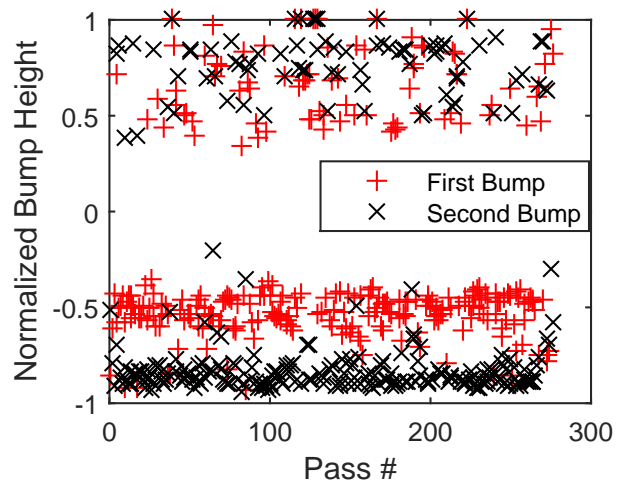

(a)

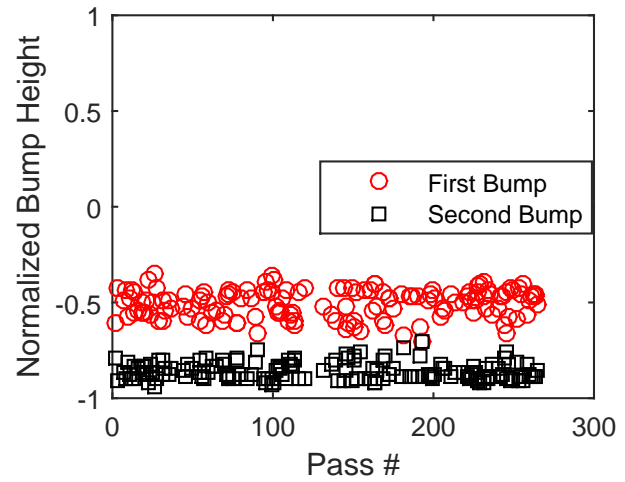

(b)

Fig. 7: Cleaning the data using the found bump heights. (a) The bump heights for the 267 passes over the $50 \mathrm{~m}$ section of track. (b) Selected passes in which the first bump is negative and the second bump is more negative than the first.

is discussed in detail at the end of this section.

One benefit of using an explicit model, such as the sparse approach, is that the train's variable speed is handled automatically by the way in which the problem is formulated. The success of the sparse approach in approximating signals from passes of different speeds is shown in Fig. 8. In Fig. 8a, two passes are shown, one where the train's average speed is $7.8 \mathrm{~m} / \mathrm{s}$ and another where the average speed is $5.4 \mathrm{~m} / \mathrm{s}$. Note that the slow pass appears to have more high-frequency content. This is because the data is sampled at a constant rate in time $(1.6 \mathrm{kHz})$, so slower passes have more samples per meter, and thus the appearance of more high frequency noise when plotted spatially. Fig. 8b shows the sparse approach applied to the two passes; in both cases, the sparse approximations model similar components of the raw signals.

Fig. 8c showcases the flexibility of the sparse approach in modeling different speeds. When the train hits a bump in the tracks, the duration of each oscillation is relatively constant and depends largely on the natural frequency and damping of the suspension. This physical intuition is clearly seen in the bottom part of Fig. 8c where the impulse responses of the systems found in analyzing the data are similar. However, in the spatial domain, the track distance covered during each oscillation depends on the train's speed, so the difference seen in the top panel of Fig. 8c is expected.

The success of the sparse method in making sense of noisy data is evident in Fig. 9. The 145 passes which conform to the pattern of interest are shown in Fig. 9a according to their recorded GPS position. As can be seen, this raw signal looks unintelligible. The sparse approximation is then found for each, and aligned according to the position of the bumps as shown in Fig. 9b. This sparse approximation reveals underlying meaning in the noisy signals: the train gets excited by two bumps along the track as it travels. Finally, we can use the information from the bumps to align the original data, which is shown in Fig. 9c.

In this particular section of track, the pattern we observe (two negative bumps) appears to occur throughout the monitoring period. This can be seen in Fig. 7, where the pass 


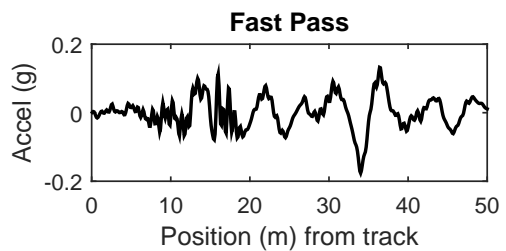

Slow Pass

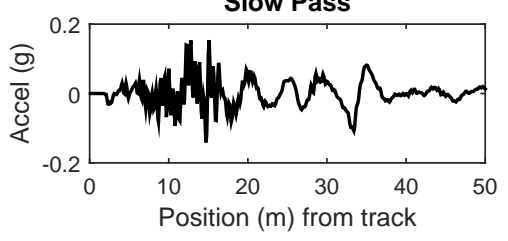

(a)

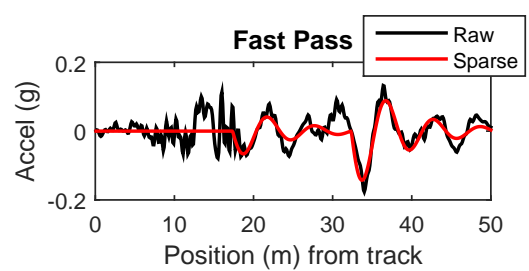

Slow Pass

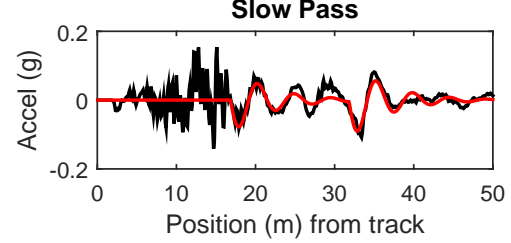

(b)

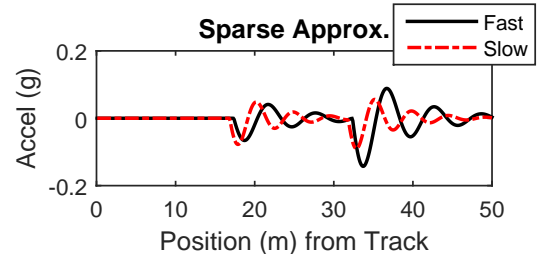

Impulse Response

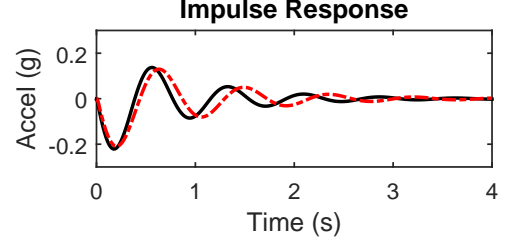

(c)

Fig. 8: The sparse approach applied to signals of different speeds, one where the train is moving at $7.8 \mathrm{~m} / \mathrm{s}$ ("Fast Pass") and one at $5.4 \mathrm{~m} / \mathrm{s}$ ("Slow Pass"). (a) The raw signals of each pass. (b) The raw signals with the sparse approximation overlaid. (c)(top) The sparse approximation for the two speeds overlaid. (c)(bottom) The impulse response of the system used for the sparse approximation. This Fig. shows that the sparse approximation correctly handles variable speed. When two sparse approximations for different speeds are shown versus position, the oscillations are different because they conform to the observed data. However the impulse response for the two approximation are similar in the time domain, showing that the system of the train does not change much with speed (as expected).

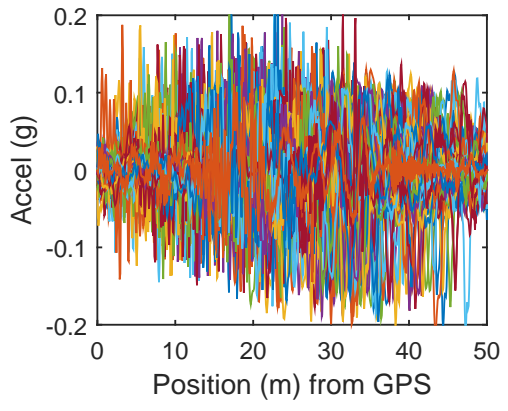

(a)

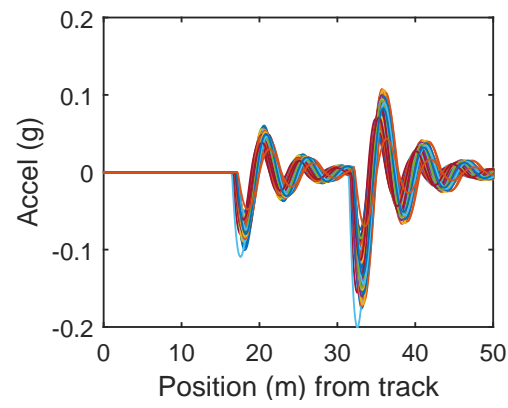

(b)

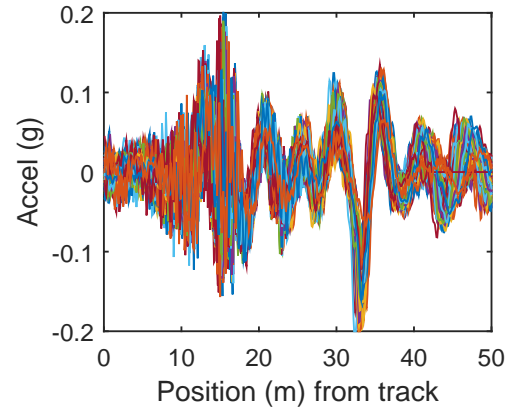

(c)

Fig. 9: The sparse approach's ability to make sense of noisy data. (a) The raw signal for the 145 passes which conform to the patten in Fig. 7b. (b) The sparse approximation for these 145 passes, aligned according to the bumps. (c) The original data from (a) now aligned according to the sparse bumps. 
indices are arranged chronologically, and the 145 selected indices are evenly distributed throughout the 267 total indices. We can confirm that for this particular $50 \mathrm{~m}$ section of track, no maintenance work was performed or requested by track inspectors according to the maintenance logs. Thus we assume that the consistency of the pattern over the 16-month period indicates that the tracks did not change significantly. A section of track that did change will be the focus of the next section, which can be detected through inconsistent patterns in bump height (as will be shown in Fig. 13).

\subsection{Detecting Track Change with the Sparse Approach}

In this section, we attempt to detect a change in track geometry using the sparse approach. The type of change here has to do with a maintenance procedure called tamping, in which the ballast underneath the tracks is adjusted to improve track geometry.

Although detecting deterioration is our ultimate goal, maintenance serves as a good proxy; deterioration is the transition from a state of good-repair to a state of mis-repair, and maintenance is the reverse transition. Directly detecting deterioration as it occurs in an operational rail system is very difficult to validate. To our knowledge, no authors have done this. During the course of our study, there were sections of track where we saw changes we believe were associated with deterioration. Unless visual inspectors had monitored that section of track daily over the period of deterioration, we could not confirm that what we had observed was in fact deterioration. The visual inspection reports of the track are conducted far less often, and due to their subjective nature, it is hard to pinpoint the state of the tracks.

Instead for this paper, we chose to validate our approach using maintenance data. These are changes to the track that we knew happened; we could cross-reference them with the maintenance logs.

In September of 2014, maintenance workers tamped the ballasted tracks on both sides of a bridge structure shown in Fig. 10. The vibration data from this change was analyzed previously using the energy in the vibration signal by our group [6], and representative results are reproduced in Fig. 11. Unlike the sparse approach, which models the track state explicitly, the signal-energy approach models the track implicitly. Although there is less energy in the signal after the tracks are tamped (i.e. the train ride is smoother), it is unclear exactly what happened. Furthermore because the track itself is not modeled, it is more challenging to align data given the position uncertainty from the GPS signal.

We next apply the sparse approach to the vibration data collected in the instrumented car from February 2014 to June 2015 around the bridge of interest. Four example passes of the raw signal are shown in Fig. 12a. The tamping activities occurred in late July 2014; two passes prior to tamping and two passes after tamping are shown. Fig. 12b shows the sparse approximation, which clearly exhibits two changes in the signal. While in the first two passes, the oscillation due to the first bump is positive, in the second two passes, it is negative. The second change can be seen towards the end of the signal: the first two passes have an additional excitation after the second major bump, while the second two do not.

We can use the height of sparse bumps as a measure of the track height. As shown in Fig. 13, if we look only at the height of the first bump, it changes dramatically after the 


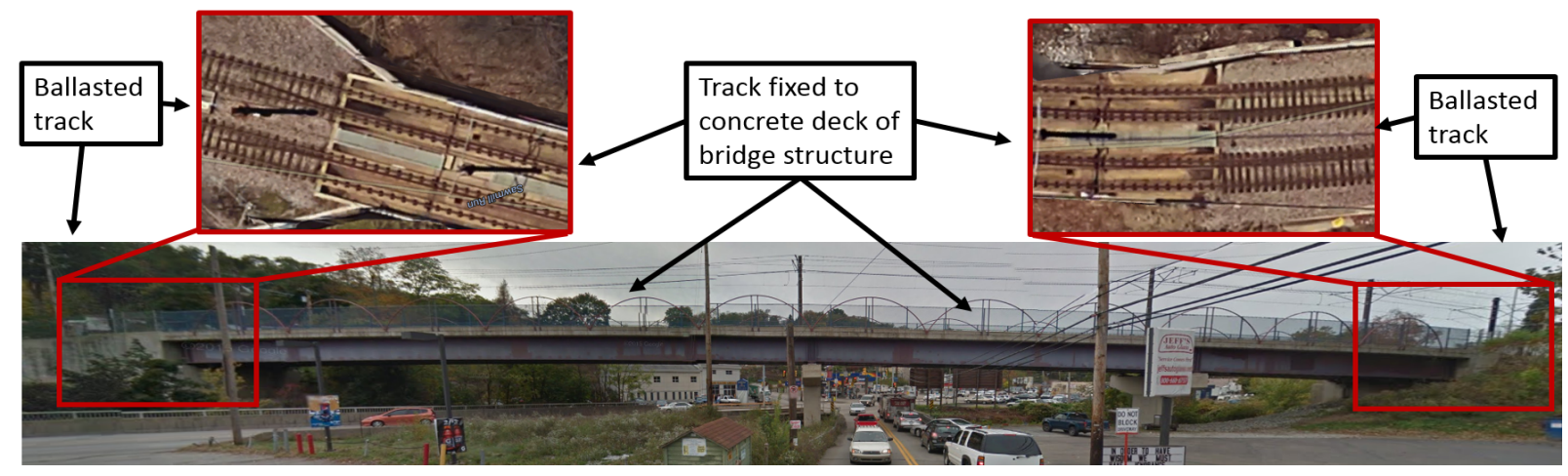

Fig. 10: The tracks and bridge of interest. The lower photo shows the entire span of the bridge, while the photos above are aerial shots showing the transition between the concrete deck and the ballasted track.

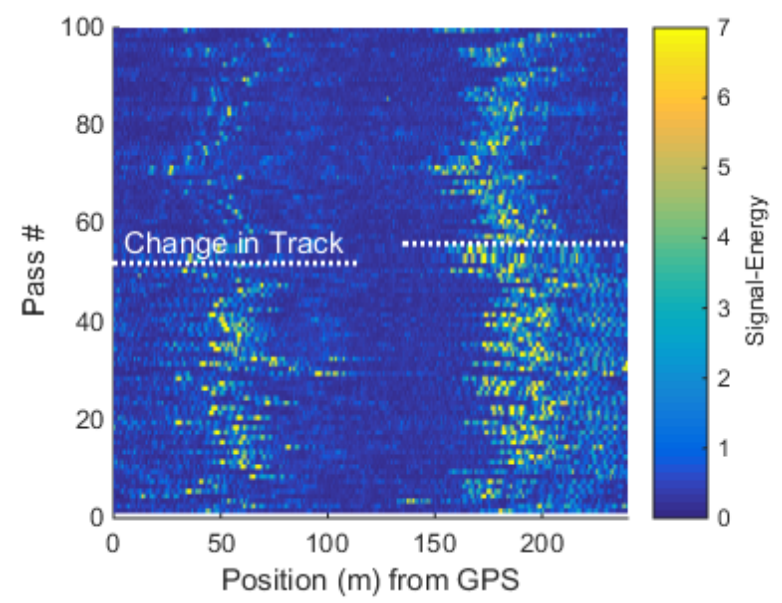

Fig. 11: The change due to tamping shown using a signal-energy feature [6]. Each horizontal line corresponds to one pass of the train over this section of track, with the color indicating the signal-energy at that location. The high energy point around $60 \mathrm{~m}$ is where the bridge starts and the high point at $180 \mathrm{~m}$ is where the bridge ends. After pass 50, the ballasted track to the left of the bridge is tamped. After pass 55, the ballasted track to the right of the bridge is tamped. Note the position here is from the GPS signal, so consecutive passes are not well aligned. 


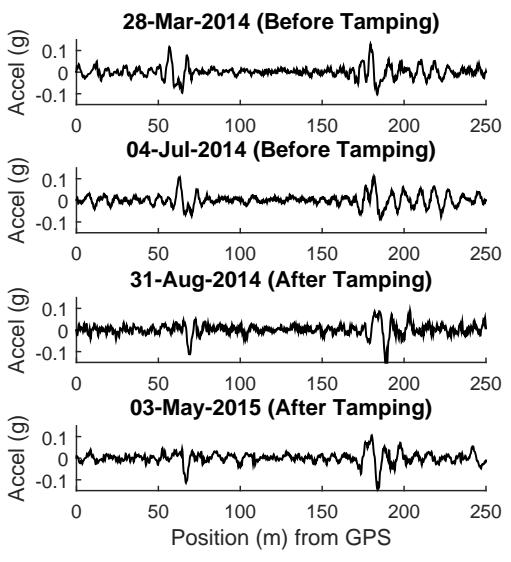

(a)

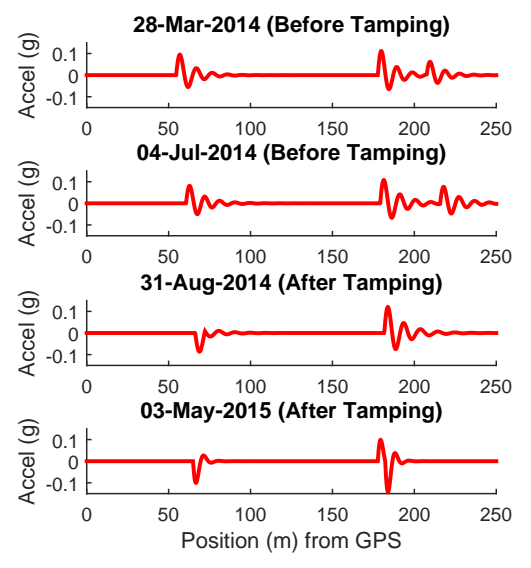

(b)

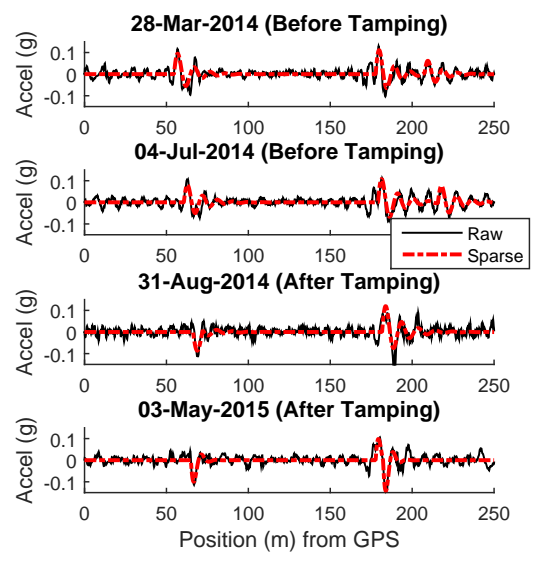

(c)

Fig. 12: The sparse approach applied to the bridge data. (a) The raw signal for two passes before tamping and two passes after tamping. Notice the train's response to the track joint at the beginning of the bridge around $60 \mathrm{~m}$ and at the end of the bridge around $180 \mathrm{~m}$ as was also seen in Fig. 11. (b) The sparse approximations for these passes. Notice that the first oscillation is positive for the first two passes and negative for the second two. (c) Comparison of the raw signal and the sparse approximation.

tamping has occurred. If the goal was to classify the state of the track, (i.e. pre-tamping versus post-tamping) this could be done simply using the sign $( \pm)$ of the first bump. In this case of the first 59 passes, 57 would be correctly identified as pre-tamping ( $96 \%$ accuracy) and of the 108 remaining passes, 98 would be identified as post-tamping (90\% accuracy). Overall, this would lead to an accuracy of $92 \%$ using just the height of one bump and a hard threshold; this is higher than the accuracy found for classifying the state of the track in [6] which was $90 \%$ using data from a $500 \mathrm{~m}$ section of track and a more sophisticated support vector machine classifier (SVM).

One remarkable aspect of the sparse approach is its ability to overcome the position uncertainty from GPS error. In previous work, a high-dimensional feature was used from each pass: the signal energy at each point along the track. Longer feature vectors are required because the position is not known exactly. Using the sparse approach, a one-dimensional feature can be used, as it consistently models the same point in the signal despite the GPS error. Because of the simplicity of the feature, a threshold can be used successfully for the classification.

However, the real power of the sparse approach is not simply its ability to achieve high classification accuracy; it is its ability to provide insight into how the track has changed. The change from positive oscillations to negative oscillations as the train enters the bridge means that the relative heights of the track around the joint have changed. Before the tamping, the train would accelerate upward as it crossed the joint, meaning the track on the bridge side of the joint was likely higher than the track on the ballasted side. After the tamping, the train would accelerate downwards meaning the track on the ballasted side is likely higher now than the track on the bridge side. This type of information could be useful 


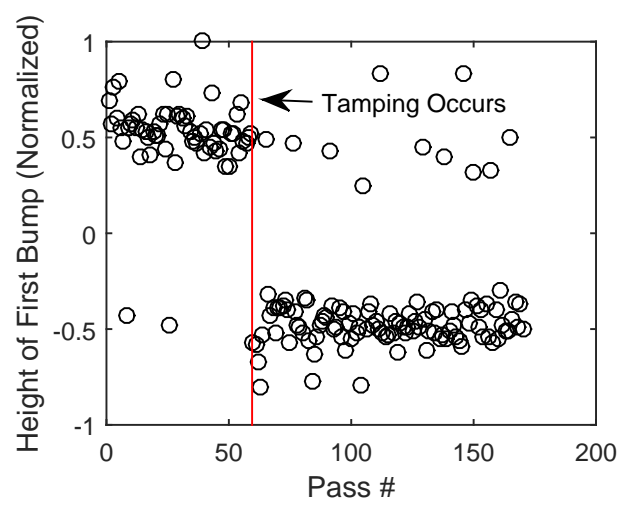

Fig. 13: The height of the first bump for each pass over this section of track. Tamping occurs after the 59 th pass. The mean of bump heights changes from 0.5 to -0.5 . This can be used as a feature to detect the change.

to inspectors. If this data were observed outside of a period of maintenance, it could signify, for example, that the bridge had settled. Such is the benefit of building an explicit model: the bumps, although sparse, directly relate to the shape of the track that most excites the train.

\section{Concluding Remarks}

In this paper we have proposed a novel sparse approach for analyzing vibration data collected from an operational train. The approach uses an iterative method to find an approximation for the track roughness and for the properties of the train. By enforcing sparsity in modeling the train system, we solve for the parameters of the train's main suspension. By enforcing sparsity in the track profile, we learn where in the tracks the train is most excited.

Applying this approach to operational train data, we show that the sparse approach can find consistent patterns in the train's response, effectively denoising the data. The identified sparse track roughness is invariant to train speed, and the location of the bumps can be used to determine the train's location. In this regard, the sparse approach helps to overcome error in the GPS signal. Furthermore, the magnitude and location of the found bumps are strong indicators of the state of the tracks, which we have shown both in cases where the track did not change, and where there was a change. In the example shown in this paper, the change detected is due to maintenance work so that the findings can be validated against work logs. We hypothesize the methods could equally be applied to detect damage, although we have not yet demonstrated this capability.

In this paper we approximate the train's fundamental mode when solving the inverse problem. Future work should look at solving for additional modes, by modeling the train as a multi-degree-of-freedom system. In addition, more accurate representation of the track could be achieved by relaxing the sparsity conditions to allow for more non-zero values in approximating the track. However, this would require a method for determining the optimal number of bumps, along with techniques to prevent over-fitting. 
Overall, the sparse approach is a promising new method for decomposing vehicle-based vibration data. We believe this technique will help enable infrastructure monitoring from in-service vehicles, making continuous objective track inspection reliable and economical.

\section{Acknowledgements}

This material is based on work supported by the National Science Foundation through a Graduate Research Fellowship for Lederman under Grant No. 0946825, by National Science Foundation awards 1130616 and 1017278, and a University Transportation Center grant (DTRT12-G-UTC11) from the US Department of Transportation. The authors also gratefully acknowledge the Port Authority of Allegheny County for their partnership, particularly David Kramer, and the many helpful workers of Amalgamated Transit Union Local 85.

\section{References}

[1] C. Esveld, Modern Railway Track: Digital Edition, 3rd Edition, MRT-Productions, 2015.

[2] D. Barke, W. K. Chiu, Structural Health Monitoring in the Railway Industry: A Review, Structural Health Monitoring 4 (1) (2005) 81-93. doi:10.1177/1475921705049764. URL http://shm. sagepub.com/content/4/1/81

[3] M. Bocciolone, A. Caprioli, A. Cigada, A. Collina, A measurement system for quick rail inspection and effective track maintenance strategy, Mechanical Systems and Signal Processing 21 (3) (2007) 12421254. doi:10.1016/j.ymssp.2006.02.007. URL http://www.sciencedirect.com/science/article/pii/S0888327006000434

[4] M. Molodova, Z. Li, R. Dollevoet, Axle box acceleration: Measurement and simulation for detection of short track defects, Wear 271 (1-2) (2011) 349-356. doi:10.1016/j.wear.2010.10.003. URL http://www.sciencedirect.com/science/article/pii/S0043164810003376

[5] P. Weston, C. Ling, C. Goodman, C. Roberts, P. Li, R. Goodall, Monitoring lateral track irregularity from in-service railway vehicles, Proceedings of the Institution of Mechanical Engineers, Part F: Journal of Rail and Rapid Transit 221 (1) (2007) 89-100.

[6] G. Lederman, S. Chen, J. Garrett, J. Kovačević, H. Noh, J. Bielak, Rail-monitoring from the dynamic response of an operational train, Journal of Mechanical Systems and Signal Processing.

[7] F. Cerda, S. Chen, J. Bielak, J. H. Garrett, P. Rizzo, J. Kovačević, Indirect structural health monitoring of a simplified laboratory-scale bridge model, Smart Structures and Systems 13 (5) (2014) 859-868.

[8] C. P. Ward, P. Weston, E. Stewart, H. Li, R. M. Goodall, C. Roberts, T. Mei, G. Charles, R. Dixon, Condition monitoring opportunities using vehicle-based sensors, Proceedings of the Institution of Mechanical Engineers, Part F: Journal of Rail and Rapid Transit 225 (2) (2011) 202-218.

[9] G. Carr, C. Diaz, J. Bloom, Method and apparatus for track geometry measurement, US Patent 6,634,112 (Oct. 21 2003). URL http://www.google.com/patents/US6634112

[10] H. A. Toliyat, K. Abbaszadeh, M. M. Rahimian, L. E. Olson, Rail defect diagnosis using wavelet packet decomposition, IEEE Transactions on Industry Applications 39 (5) (2003) 1454-1461.

[11] S. Hensel, C. Hasberg, C. Stiller, Probabilistic rail vehicle localization with eddy current sensors in topological maps, IEEE Transactions on Intelligent Transportation Systems 12 (4) (2011) 1525-1536.

[12] A. Caprioli, A. Cigada, D. Raveglia, Rail inspection in track maintenance: A benchmark between the wavelet approach and the more conventional Fourier analysis, Mechanical Systems and Signal Processing 21 (2) (2007) 631-652. doi:10.1016/j.ymssp.2005.12.001. URL http://www.sciencedirect.com/science/article/pii/S0888327005002402

[13] Y. Naganuma, M. Kobayashi, M. Nakagawa, T. Okumura, Condition monitoring of shinkansen tracks using commercial trains, in: 2008 4th IET International Conference on Railway Condition Monitoring, 2008, pp. 1-6. 
[14] Y. Oshima, K. Yamamoto, K. Sugiura, A. Tanaka, M. Hori, Simultaneous monitoring of the coupled vibration between a bridge and moving trains, in: Proc. 5th IABMAS Conference, Philadelphia, USA, 2010, p. 186.

URL http://www.crcnetbase.com/doi/abs/10.1201/b10430-114

[15] J. Real, P. Salvador, L. Montalbán, M. Bueno, Determination of Rail Vertical Profile through Inertial Methods, Proceedings of the Institution of Mechanical Engineers, Part F: Journal of Rail and Rapid Transit 225 (1) (2011) 14-23. doi:10.1243/09544097JRRT353. URL http://pif . sagepub.com/content/225/1/14

[16] M. Molodova, Z. Li, A. Nunez, R. Dollevoet, Automatic Detection of Squats in Railway Infrastructure, IEEE Transactions on Intelligent Transportation Systems 15 (5) (2014) 1980-1990. doi:10.1109/TITS.2014.2307955.

[17] J. S. Lee, S. Choi, S.-S. Kim, C. Park, Y. G. Kim, A mixed filtering approach for track condition monitoring using accelerometers on the axle box and bogie, IEEE Transactions on Instrumentation and Measurement 61 (3) (2012) 749-758.

[18] E. J. O'Brien, C. Bowe, P. Quirke, Determination of vertical alignment of track using accelerometer readings, in: IMechE Stephenson Conference for Railways: Research for Railways, 21-23 April, 2015, 2015. URL http://researchrepository.ucd.ie/handle/10197/7000

[19] O. Heirich, A. Lehner, P. Robertson, T. Strang, Measurement and analysis of train motion and railway track characteristics with inertial sensors, in: 14th International IEEE Conference on Intelligent Transportation Systems (ITSC), IEEE, 2011, pp. 1995-2000.

[20] G. Lederman, Z. Wang, J. Bielak, H. Noh, J. Garrett, S. Chen, J. Kovačević, F. Cerda, P. Rizzo, Damage quantification and localization algorithms for indirect shm of bridges, in: 7th International Conference on Bridge Maintenance, Safety and Management (IABMAS), 2014, pp. 640-647. doi:10.1201/b1706393.

[21] P. Weston, C. Ling, C. Roberts, C. Goodman, P. Li, R. Goodall, Monitoring vertical track irregularity from in-service railway vehicles, Proceedings of the institution of mechanical engineers, Part F: Journal of Rail and Rapid Transit 221 (1) (2007) 75-88.

[22] D. Cantero, B. Basu, Railway infrastructure damage detection using wavelet transformed acceleration response of traversing vehicle, Structural Control and Health Monitoring 22 (1) (2015) 62-70.

[23] S. Chen, F. Cerda, P. Rizzo, J. Bielak, J. H. Garrett, J. Kovačević, Semi-supervised multiresolution classification using adaptive graph filtering with application to indirect bridge structural health monitoring, IEEE Transactions on Signal Processing 62 (11) (2014) 2879-2893.

[24] G. Lederman, H. Noh, J. Bielak, Rail-infrastructure monitoring through the dynamic response of a passing train, in: International Workshop on Structural Health Monitoring, Stanford, CA. Sep 1-3, 2015. doi:10.12783/SHM2015/182.

[25] Y. Yang, S. Nagarajaiah, Structural damage identification via a combination of blind feature extraction and sparse representation classification, Mechanical Systems and Signal Processing 45 (1) (2014) 1-23.

[26] H. Noh, A. S. Kiremidjian, Application of a sparse representation method using k-svd to data compression of experimental ambient vibration data for shm, in: SPIE Smart Structures and Materials+ Nondestructive Evaluation and Health Monitoring, International Society for Optics and Photonics, 2011, pp. $79814 \mathrm{~N}-79814 \mathrm{~N}$.

[27] M. Eybpoosh, M. Berges, H. Noh, Sparse representation of ultrasonic guided-waves for robust damage detection in pipelines under varying environmental and operational conditions, Structural Control and Health Monitoring 23 (2) (2016) 369-391.

[28] Z. Wang, S. Chen, G. Lederman, F. Cerda, J. Bielak, J. Garrett, P. Rizzo, J. Kovačević, Comparison of sparse representation and fourier discriminant methods: Damage location classification in indirect lab-scale bridge structural health monitoring, in: The 2013 Structures Congress, 2013, pp. 436-446.

[29] S. S. Saab, A map matching approach for train positioning part I: development and analysis, IEEE Transactions on Vehicular Technology 49 (2) (2000) 467-475.

[30] O. Heirich, P. Robertson, T. Strang, Railslam-localization of rail vehicles and mapping of geometric 
railway tracks, in: 2013 IEEE International Conference on Robotics and Automation (ICRA), IEEE, 2013, pp. 5212-5219.

[31] E. G. Berggren, M. X. D. Li, J. Spännar, A new approach to the analysis and presentation of vertical track geometry quality and rail roughness, Wear 265 (9-10) (2008) 1488-1496. doi:10.1016/j.wear.2008.01.029. URL http://www.sciencedirect.com/science/article/pii/S0043164808001828

[32] A. P. Dempster, N. M. Laird, D. B. Rubin, Maximum likelihood from incomplete data via the EM algorithm, Journal of the royal statistical society. Series B (methodological) (1977) 1-38.

[33] D. Ge, X. Jiang, Y. Ye, A note on the complexity of L p minimization, Mathematical programming 129 (2) (2011) 285-299.

[34] S. G. Mallat, Z. Zhang, Matching pursuits with time-frequency dictionaries, IEEE Transactions on Signal Processing 41 (12) (1993) 3397-3415.

[35] M. Vetterli, J. Kovačević, V. K. Goyal, Foundations of Signal Processing, Cambridge University Press, 2014.

[36] M. Dumitriu, Influence of the Suspension Damping on the Ride Comfort of Passenger Railway Vehicles, Scientific Bulletin University Plitechnica of Bucharest Series D 74 (4).

[37] G. Lederman, J. Bielak, H. Noh, Rail monitoring from the dynamic response of a passenger train, Structural Health Monitoring Applications Case Study Archive. URL https://sites.tufts.edu/shmcasestudies/

[38] K. Aki, B. Chouet, Origin of coda waves: source, attenuation, and scattering effects, Journal of geophysical research 80 (23) (1975) 3322-3342. 